\title{
An Ultralocal Classical and Quantum Gravity Theory
}

\author{
John R. Klauder \\ Department of Physics and Department of Mathematics, University of Florida, Gainesville, FL, USA \\ Email: klauder@phys.ufl.edu
}

How to cite this paper: Klauder, J.R. (2020) An Ultralocal Classical and Quantum Gravity Theory. Journal of High Energy Physics, Gravitation and Cosmology, 6, 656661.

https://doi.org/10.4236/jhepgc.2020.64044

Received: July 4, 2020

Accepted: September 6, 2020

Published: September 9, 2020

Copyright (C) 2020 by author(s) and Scientific Research Publishing Inc.

This work is licensed under the Creative Commons Attribution International License (CC BY 4.0)

http://creativecommons.org/licenses/by/4.0/

\section{Open Access}

\begin{abstract}
An ultralocal form of any classical field theory eliminates all spatial derivatives in its action functional, e.g., in its Hamiltonian functional density. It has been applied to covariant scalar field theories and even to Einstein's general relativity, by Pilati, as an initial term in a perturbation series that aimed to restore all omitted derivatives. Previously, the author has quantized ultralocal scalar fields by affine quantization to show that these non-renormalizanle theories can be correctly quantized by affine quantization; the story of such scalar models is discussed in this paper. The present paper will also show that ultralocal gravity can be successfully quantized by affine quantization. The purpose of this study is that a successful affine quantization of any ultralocal field problem implies that, with properly restored derivatives, the classical theory can, in principle, be guaranteed a successful result using either a canonical quantization or an affine quantization. In particular, Einstein's gravity requires an affine quantization, and it will be successful.
\end{abstract}

\section{Keywords}

Cosmological Constant, Ultralocal Models, Affine Quantization

\section{Ultralocal Scalar Field Theories}

Before considering gravity, it can be useful to review a modest summary of the results of canonical quantization when it has been used to study a variety of covariant scalar field models.

A traditional covariant scalar field has a classical (c) Hamiltonian given by

$$
H_{c}=\int\left\{\frac{1}{2}\left[\pi(x)^{2}+(\vec{\nabla} \varphi)(x)^{2}+m_{0}^{2} \varphi(x)^{2}\right]+g_{0} \varphi(x)^{p}\right\} d^{s} x,
$$

with $p$ an even positive integer, $s$, the number of spacial dimensions, is a positive integer, $m_{0}^{2}>0$, and $g_{0} \geq 0$. With $n=s+1$ spacetime dimensions and using canonical quantization, a satisfactory quantization appears for $p<2 n /(n-2)$. If $p \geq 4=n$ a Monte Carlo study [1] 
and analysis [2,3] only found "free theory results", while if perturbation series were used when $p>2 n /(n-2)$, then only, divergent, non-renormalizable behavior appeared. The ultralocal $(u)$ scalar field model here has a classical Hamiltonian given by

$$
H_{u}=\int\left\{\frac{1}{2}\left[\pi(x)^{2}+m_{0}^{2} \varphi(x)^{2}\right]+g_{0} \varphi(x)^{p}\right\} d^{s} x,
$$

and with canonical quantization, a divergent perturbation series for $p>2$ and any $n \geq 2$ leads to undesired results. Let us choose a different path.

The domain of $H_{u}$ consists of all, continuous momentum functions $\pi(x)$ and scalar fields $\varphi(x)$ for which $0<H_{u}<\infty$. Our interest is focussed on $p>2$, and thus $p \in\{4,6,8, \ldots\}$, and clearly, for all such $p>2$ and all $s \in\{1,2,3, \ldots\}$, the domain $\mathcal{D}_{p>2}(\pi, \varphi) \subset \mathcal{D}_{p=2}(\pi, \varphi)$ we will raise the issue of domains again at the end of this story. When quantized by using canonical quantization, along with a perturbation series in powers of $g_{0}$, one encounters multiple infinities.

Since all derivatives have been removed, what happens at any point of $x$ does not affect what happens at any other point $x^{\prime} \neq x$. Just like sums of independent operators in Hamiltonians leads to products of separate and independent wave functions, this implies, for our problem, that the ground state is composed of products at different points, which - thanks to the Central Limit Theorem - leads to the characteristic function of the ground state distribution, i.e., the Fourier transformation of the absolute square of the normalized ground state, being given in the form of

$$
\begin{aligned}
C(f) & =\int e^{i \int f(x) \varphi(x) d^{s} x} \Pi_{x}\left|\Psi_{0}[\varphi(x)]\right|^{2} d \varphi(x) \\
& =e^{-\int W(f(x)) d^{s} x}
\end{aligned}
$$

for a suitable function $W(f(x))$, with $W(f(x))=0$ whenever $f(x)=0$.

\subsection{An Affine Ultralocal Scalar Field}

Affine classical variables are given by $\kappa(x) \equiv \pi(x) \varphi(x)$ and $\varphi(x)$, with the restriction that $\varphi(x) \neq 0$, and the Poisson bracket is given by $\left\{\varphi(x), \kappa\left(x^{\prime}\right)\right\}=\delta^{s}\left(x-x^{\prime}\right) \varphi(x)$. The classical ultralocal Hamiltonian expressed in affine variables is given by

$$
H_{u}=\int\left\{\frac{1}{2}\left[\kappa(x)^{2} \varphi(x)^{-2}+m_{0}^{2} \varphi(x)^{2}\right]+g_{0} \varphi(x)^{p}\right\} d^{s} x .
$$

The basic quantum operators are $\hat{\varphi}(x) \neq 0$ and $\hat{\kappa}(x)$, and their commutator is given by $\left[\hat{\varphi}(x), \hat{\kappa}\left(x^{\prime}\right)\right]=i \hbar \delta^{s}\left(x-x^{\prime}\right) \hat{\varphi}(x)$. The quantum affine Hamiltonian is given by

$$
\mathcal{H}_{u}=\int\left\{\frac{1}{2}\left[\hat{\kappa}(x) \hat{\varphi}(x)^{-2} \hat{\kappa}(x)+m_{0}^{2} \hat{\varphi}(x)^{2}\right]+g_{0} \hat{\varphi}(x)^{p}\right\} d^{s} x,
$$

and the Schrödinger representation is given by $\hat{\varphi}(x)=\varphi(x)$ and

$$
\left.\hat{\kappa}(x)=-\frac{1}{2} i \hbar[\varphi(x)(\delta / \delta \varphi(x)))+(\delta / \delta \varphi(x)) \varphi(x)\right] .
$$

Clearly, this is a formal equation for the Hamiltonian operator, etc. Such expressions deserve a regularization of these equations.

\subsection{A Regularized Affine Ultralocal Scalar Field}

Our regularization is of the underlying space in which $x \rightarrow \mathbf{k} a$, where $\mathbf{k} \in\{\ldots,-1,0,1,2,3, \ldots\}^{s}$ and $a>0$ denotes the tiny distance between 
lattice rungs. The regularized classical ultralocal Hamiltonian is given by

$$
H_{u}=\sum_{\mathbf{k}}\left\{\frac{1}{2}\left[\pi_{\mathbf{k}}^{2}+m_{0}^{2} \varphi_{\mathbf{k}}^{2}\right]+g_{0} \varphi_{\mathbf{k}}^{p}\right\} a^{s} .
$$

The classical affine regularization involves $\kappa_{\mathbf{k}}=\pi_{\mathbf{k}} \varphi_{\mathbf{k}}$ and $\varphi_{\mathbf{k}}$, with $\varphi_{\mathbf{k}} \neq 0$, with a Poisson bracket $\left\{\varphi_{\mathbf{k}}, \kappa_{\mathbf{m}}\right\}=\delta_{\mathbf{k}, \mathbf{m}} \varphi_{\mathbf{k}}$, and the classical affine regularized ultralocal Hamiltonian is given by

$$
\mathcal{H}_{u}=\sum_{\mathbf{k}}\left\{\frac{1}{2}\left[\kappa_{\mathbf{k}}^{2} \varphi_{\mathbf{k}}^{-2}+m_{0}^{2} \varphi_{\mathbf{k}}^{2}\right]+g_{0} \varphi_{\mathbf{k}}^{p}\right\} a^{s} .
$$

The regularized basic quantum Schrödinger operators are given by $\hat{\varphi}_{\mathbf{k}}=\varphi_{\mathbf{k}}$ and

$$
\begin{aligned}
\hat{\kappa}_{\mathbf{k}} & =-i \frac{1}{2} \hbar\left[\varphi_{\mathbf{k}}\left(\partial / \partial \varphi_{\mathbf{k}}\right)+\left(\partial / \partial \varphi_{\mathbf{k}}\right) \varphi_{\mathbf{k}}\right] a^{-s} \\
& =-i \hbar\left[\varphi_{\mathbf{k}}\left(\partial / \partial \varphi_{\mathbf{k}}\right)+1 / 2\right] a^{-s} .
\end{aligned}
$$

An important result is that $\hat{\kappa}_{\mathbf{k}} \varphi_{\mathbf{k}}^{-1 / 2}=0$. The Schrödinger equation becomes

$$
i \hbar \partial \psi(\varphi, t) / \partial t=\sum_{\mathbf{k}}\left\{\frac{1}{2}\left[\hat{\kappa}_{\mathbf{k}} \varphi_{\mathbf{k}}^{-2} \hat{\kappa}_{\mathbf{k}}+m_{0}^{2} \varphi_{\mathbf{k}}^{2}\right]+g_{0} \varphi_{\mathbf{k}}^{p}\right\} a^{s} \psi(\varphi, t) .
$$

The normalized ground state of such an equation is given by

$$
\psi_{0}(\varphi)=\Pi_{\mathbf{k}} e^{-V\left(\varphi_{\mathbf{k}}\right) / 2}\left(b a^{s}\right)^{1 / 2} \varphi_{\mathbf{k}}^{-\left(1-2 b a^{s}\right) / 2},
$$

for some real function $V\left(\varphi_{\mathbf{k}}\right)$. Finally, we ask what is the characteristic function for such an equation, and the answer is given by

$$
\begin{aligned}
C(f) & =\lim _{a \rightarrow 0} \Pi_{\mathbf{k}} \int e^{i f_{\mathbf{k}} \varphi_{\mathbf{k}}} e^{-V\left(\varphi_{\mathbf{k}}\right)}\left(b a^{s}\right)\left|\varphi_{\mathbf{k}}\right|^{-\left(1-2 b a^{s}\right)} d \varphi_{\mathbf{k}} \\
& =\lim _{a \rightarrow 0} \Pi_{\mathbf{k}}\left[1-\left(b a^{s}\right) \int\left[1-e^{i f_{\mathbf{k}} \varphi_{\mathbf{k}}}\right] e^{-V\left(\varphi_{\mathbf{k}}\right)}\left|\varphi_{\mathbf{k}}\right|^{-\left(1-2 b a^{s}\right)} d \varphi_{\mathbf{k}}\right. \\
& =\exp \left\{-b \int d^{s} x \int\left[1-e^{i f(x) \lambda}\right] e^{-v(\lambda)} d \lambda /|\lambda|\right\} .
\end{aligned}
$$

Here $\varphi_{\mathbf{k}} \rightarrow \lambda$, and $V \rightarrow v$ to account for changes that may have arisen in $V$ as $a \rightarrow 0$. The resultant expression in (12) is a (generalized) Poisson distribution, which, besides a Gaussian distribution, is the only other form allowed by the Central Limit Theorem.

As a crude estimate of the large behavior of $\varphi$ for different $p$ values we can examine $\left(-d^{2} / d x^{2}+x^{p}\right) e^{-|x|^{\gamma} / \gamma} \simeq\left(-x^{2 \gamma-2}+x^{p}+\cdots\right) e^{-|x|^{\gamma / \gamma}}$. To cancel the largest terms we roughly need to have $\gamma=1+p / 2$. If $g_{0} \rightarrow 0$, then $p \rightarrow 2=\gamma$, and thus $v(\lambda) \rightarrow c \lambda^{2}$, with $c>0$. This last fact means that sending $g_{0} \rightarrow 0$ does not lead us to a simple, free Gaussian because the given result reflects the continuity of a smaller domain of the interacting model as compared to the strictly larger domain of the truly free theory.

\section{The Main Lesson from Ultralocal Scalar Fields}

The previous subsection found that an ultralocal scalar field model led to acceptable results when $p \geq 2$ and $n \geq 2$. For certain covariant scalar field models, we have already observed that acceptable results arise by canonical quantization when $p<2 n /(n-2)$. In view of acceptable results for ultralocal scalar fields when $p \geq 2$ and $n \geq 2$, we predict that an affine quantization for covariant scalar fields leads to acceptable results when $p \geq 2 n /(n-2)$. Monte Carlo studies, such as those carried out in [1], could confirm whether this prediction is true or not. 


\section{Ultralocal General Relativity}

An effort to quantize Einstein's theory of gravity has been examined in several articles published by the author; see [4-7], with [8], perhaps, being the strongest effort of them all. In light of those articles, we will present a modest selection of the necessary features for an affine quantization of Einstein's gravity.

The phase space variables in the ADM version of classical general relativity [9] are the metric field $g_{a b}(x)$ and the momentum field $\pi^{c d}(x)$, where $a, b, c, d, \ldots=1,2,3$, and which a canonical quantization promotes to basic quantum operators. The positivity requirement that $g_{a b}(x) d x^{a} d x^{b}>0$ implies that the momentum operator can not be self adjoint. An affine quantization chooses the classical metric $g_{a b}(x)$, which has a positive requirement as before, while the momentum field is replaced by the momentric field $\pi_{b}^{a}(x)\left[\equiv \pi^{a c}(x) g_{b c}(x)\right]$. These basic affine variables are promoted to quantum operators, both of which can be self adjoint, while the metric operator is also positive as desired.

The ADM classical Hamiltonian, with $g(x) \equiv \operatorname{det}\left[g_{a b}(x)\right]>0$, is given by

$H_{c}=\int\left\{g(x)^{-1 / 2}\left[\pi_{b}^{a}(x) \pi_{a}^{b}(x)-\frac{1}{2} \pi_{a}^{a}(x) \pi_{b}^{b}(x)\right]+g(x)^{1 / 2} R(x)\right\} d^{3} x$

where $R(x)$ is the 3 -dimensional scalar curvature.

The term $R(x)$ contains all of the spatial derivative terms and the ultralocal version of the classical Hamiltonian is chosen as

$$
H_{u}=\int\left\{g(x)^{-1 / 2}\left[\pi_{b}^{a}(x) \pi_{a}^{b}(x)-\frac{1}{2} \pi_{a}^{a}(x) \pi_{b}^{b}(x)\right]+g(x)^{1 / 2} \Lambda(x)\right\} d^{3} x .
$$

This expression now has a position-dependent "cosmological constant" in place of the scalar curvature. The term $\Lambda(x)$ (imitating $R(x)$ ) should be a continuous function that obeys $-\infty<\Lambda(x)<\infty$. When this Hamitonian is quantizaed the only variables that are promoted to quantum operators are the metric field, $g_{a b}(x)$, and the momentric field, $\pi_{d}^{c}(x)$; the classical function $\Lambda(x)$ is fixed and not made into an operator. ${ }^{1}$

\subsection{An Affine Quantization of Ultralocal Gravity}

The quantum operators are $\hat{g}_{a b}(x)$ and $\hat{\pi}_{d}^{c}(x)$, and their Schrödinger representations are given by $\hat{g}_{a b}(x)=g_{a b}(x)$ and

$$
\hat{\pi}_{b}^{a}(x)=-i \frac{1}{2} \hbar\left[g_{b c}(x)\left(\delta / \delta g_{a c}(x)\right)+\left(\delta / \delta g_{a c}(x)\right) g_{b c}(x)\right] .
$$

The Schrödinger equation for the ultralocal Hamiltonian is given by

$$
\begin{aligned}
& i \hbar \partial \psi(\{g\}, t) / \partial t=\int\left\{\hat{\pi}_{b}^{a}(x) g(x)^{-1 / 2} \hat{\pi}_{a}^{b}(x)\right. \\
& \left.-\frac{1}{2} \hat{\pi}_{a}^{a}(x) g(x)^{-1 / 2} \hat{\pi}_{b}^{b}(x)+g(x)^{1 / 2} \Lambda(x)\right\} d^{3} x \psi(\{g\}, t),
\end{aligned}
$$

where the symbol $\{g\}$ denotes the full metric matrix. In addition, we find that $\hat{\pi}_{b}^{a}(x) g(x)^{-1 / 2}=0$, which is proved in [8], just above Equation (40). Solutions of (16) are again governed by the Central Limit Theorem.

As was the scalar case in the previous section, the formal expression for (16) needs to be regularized.

1 Pilati discussed a similar model [10,11] in an effort to use such a model as the first term in a perturbation series to restore the proper gravity using canonical quantization. 


\subsection{A Regularized Affine Ultralocal Quantum Gravity}

Much like the regularization of the scalar fields, we introduce a discrete version of the underlying space such as $x \rightarrow \mathbf{k} a$, where $\mathbf{k} \in$ $\{\ldots,-1,0,1,2,3, \ldots\}^{3}$ and $a>0$ is the spacing between rungs in which, for the Schrödinger representation, $g_{a b}(x) \rightarrow g_{a b \mathbf{k}}$ and $\hat{\pi}_{d}^{c}(x) \rightarrow \hat{\pi}_{d \mathbf{k}}^{c}$ that becomes

$$
\begin{aligned}
\hat{\pi}_{d \mathbf{k}}^{c} & =-i \frac{1}{2} \hbar\left[g_{d e \mathbf{k}}\left(\partial / \partial g_{c e \mathbf{k}}\right)+\left(\partial / \partial g_{c e \mathbf{k}}\right) g_{d e \mathbf{k}}\right] a^{-s} \\
& =-i \hbar\left[g_{d e \mathbf{k}}\left(\partial / \partial g_{c e \mathbf{k}}\right)+\delta_{d}^{c} / 2\right] a^{-s} .
\end{aligned}
$$

Take note that $\hat{\pi}_{b \mathbf{k}}^{a} g_{\mathbf{k}}^{-1 / 2}=0$, where $g_{\mathbf{k}}=\operatorname{det}\left(g_{a b \mathbf{k}}\right)$.

The regularized Schrödinger equation is then given by

$$
\begin{aligned}
i \hbar \partial \psi(\{g\}, t) / \partial t & =\sum_{\mathbf{k}}\left\{\hat{\pi}_{b \mathbf{k}}^{a} g_{\mathbf{k}}^{-1 / 2} \hat{\pi}_{a \mathbf{k}}^{b}-\frac{1}{2} \hat{\pi}_{a \mathbf{k}}^{a} g_{\mathbf{k}}^{-1 / 2} \hat{\pi}_{b \mathbf{k}}^{b}\right. \\
& \left.+g_{\mathbf{k}}^{1 / 2} \Lambda_{\mathbf{k}}\right\} a^{s} \psi(\{g\}, t),
\end{aligned}
$$

A normalized, stationary solution to this equation is given by

$$
\psi_{Y}(\{g\})=\Pi_{\mathbf{k}} Y\left(g_{\mathbf{k}}, \Lambda_{\mathbf{k}}\right)\left(b a^{3}\right)^{1 / 2} g_{\mathbf{k}}^{-\left(1-b a^{3}\right) / 2} .
$$

The characteristic function for such an expression is given by

$$
\begin{aligned}
C_{Y}(f) & =\lim _{a \rightarrow 0} \Pi_{\mathbf{k}} \int e^{i f_{\mathbf{k}} g_{\mathbf{k}}}\left|Y\left(g_{\mathbf{k}}, \Lambda_{\mathbf{k}}\right)\right|^{2}\left(b a^{3}\right) g_{\mathbf{k}}^{-\left(1-b a^{3}\right)} d g_{\mathbf{k}} \\
& =\lim _{a \rightarrow 0} \Pi_{\mathbf{k}}\left\{1-\left(b a^{3}\right) \int\left[1-e^{i f_{\mathbf{k}} g_{\mathbf{k}}}\right]\left|Y\left(g_{\mathbf{k}}, \Lambda_{\mathbf{k}}\right)\right|^{2} g_{\mathbf{k}}^{-\left(1-b a^{3}\right)} d g_{\mathbf{k}}\right\} \\
& =\exp \left\{-b \int d^{3} x \int\left[1-e^{i f(x) \mu}\right]|y(\mu, \Lambda(x))|^{2} d \mu / \mu\right\}
\end{aligned}
$$

where the scalar $g_{\mathbf{k}} \rightarrow \mu>0$ and $Y \rightarrow y$ to accommodate any change in $Y$ due to $a \rightarrow 0$. The final result is a (generalized) Poisson distribution, which obeys the Central Limit Theorem.

The formulation of characteristic functions for gravity establishes the suitability of affine quantization as claimed. Although this analysis was only for an ultralocal model, it nevertheless points to the existence of proper solutions for Einstein's general relativity.

\section{The Main Lesson from Ultralocal Gravity}

Just like the success of quantizing ultralocal scalar models, we have also showed that ultralocal gravity can be quantized using affine quantization. The purpose of solving ultralocal scalar models was to ensure that non-renormalizable covariant scalar fields can also be solved using affine quantization. Likewise, the purpose of quantizing an ultralocal version of Einstein's gravity shows that we should, in principle, and using affine quantization, be able to quantize the genuine version of Einstein's gravity using affine quantization.

The analysis of certain gravity models with significant symmetry may provide examples that can be completely solved using the tools of affine quantization. For readers interested in the why and the how of affine quantization, perhaps [5] could be a good place to start.

\section{Conflicts of Interest}

The author declares no conflicts of interest regarding the publication of this paper. 


\section{References}

[1] Freedman, B., Smolensky, P. and Weingarten, D. (1982) Monte Carlo Evaluation of the Continuum Limit of $\varphi_{4}^{4}$ and $\varphi_{3}^{4}$. Physics Letters B, 113, 481-486. https://doi.org/10.1016/0370-2693(82)90790-0

[2] Aizenman, M. (1981) Proof of the Triviality of $\varphi_{d}^{4}$ Field Theory and Some Mean-Field Features of Ising Models for $d>4$. Physical Review Letters, 47, 886. https://doi.org/10.1103/PhysRevLett.47.886

[3] Fröhlich, J. (1982) On the Triviality of $\lambda \varphi_{d}^{4}$ Theories and the Approach to the Critical Point in $d \geq 4$ Dimensions. Nuclear Physics B, 200, 281-296.

https://doi.org/10.1016/0550-3213(82)90088-8

[4] Klauder, J.R. (2020) Quantum Gravity Made Esay. Journal of High Energy Physics, Gravitation and Cosmology, 6, 90-102. https://doi.org/10.4236/jhepgc.2020.61009

[5] Klauder, J.R. (2020) The Benefits of Affine Quantization. Journal of High Energy Physics, Gravitation and Cosmology, 6, 175-185. https://doi.org/10.4236/jhepgc.2020.62014

[6] Klauder, J.R. (2020) Building a Genuine Quantum Gravity. Journal of High Energy Physics, Gravitation and Cosmology, 6, 159173. https://doi.org/10.4236/jhepgc.2020.61013

[7] Klauder, J.R. (2020) Quantum Gravity, Constant Negative Curvatures, and Black Holes. Journal of High Energy Physics, Gravitation and Cosmology, 6, 313-320.

https://doi.org/10.4236/jhepgc.2020.63024

[8] Klauder, J.R. (2020) Using Affine Quantization to Analyze NonRenormalizable Scalar Fields and the Quantization of Einstein's Gravity. arXiv:2006.09156

[9] Arnowitt, R., Deser, S. and Misner, C. (1962) The Dynamics of General Relativity. In: Witten, L., Ed., Gravitation: An Introduction to Current Research, Wiley \& Sons, New York, 227.

[10] Pilati, M. (1982) Strong Coupling Quantum Gravity. I. Solution in a Particular Gauge. Physical Review D, 26, 2645.

https://doi.org/10.1103/PhysRevD.26.2645

[11] Pilati, M. (1983) Strong Coupling Quantum Gravity. II. Solution without Gauge Fixing. Physical Review D, 28, 729. https://doi.org/10.1103/PhysRevD.28.729 